Published in final edited form as:

Nat Immunol. 2010 October ; 11(10): 920-927. doi:10.1038/ni.1931.

\title{
SLAM is a microbial sensor that regulates bacterial phagosome functions in macrophages
}

\author{
Scott B Berger ${ }^{1,7}$, Xavier Romero ${ }^{1,7}$, Chunyan Ma ${ }^{1}$, Guoxing Wang ${ }^{1}$, William A Faubion ${ }^{1,2}$, \\ Gongxian Liao ${ }^{1}$, Ewoud Compeer ${ }^{1}$, Marton Keszei ${ }^{1}$, Lucia Rameh ${ }^{3}$, Ninghai Wang ${ }^{1}$, \\ Marianne Boes ${ }^{4}$, Jose R Regueiro ${ }^{1,5}$, Hans-Christian Reinecker ${ }^{6}$, and Cox Terhorst ${ }^{1}$ \\ ${ }^{1}$ Division of Immunology, Beth Israel Deaconess Medical Center, Harvard Medical School, \\ Boston, Massachusetts, USA \\ ${ }^{2}$ Department of Internal Medicine, Mayo Clinic, Rochester, Minnesota, USA \\ ${ }^{3}$ Boston Biomedical Research Institute, Watertown, Massachusetts, USA \\ ${ }^{4}$ Department of Dermatology, Brigham and Women's Hospital, Harvard Medical School, Boston, \\ Massachusetts, USA \\ ${ }^{5}$ Inmunologia, Facultad de Medicina, Universidad Complutense, Madrid, Spain \\ ${ }^{6}$ Department of Medicine, Gastrointestinal Unit and Center for the Study of Inflammatory Bowel \\ Disease, Massachusetts General Hospital, Harvard Medical School, Massachusetts, USA
}

\section{Abstract}

Phagocytosis is a pivotal process by which macrophages eliminate microorganisms after recognition by pathogen sensors. Here we unexpectedly found that the self ligand and cell surface receptor SLAM functioned not only as a costimulatory molecule but also as a microbial sensor that controlled the killing of Gram-negative bacteria by macrophages. SLAM regulated activity of the NADPH oxidase NOX2 complex and phagolysosomal maturation after entering the phagosome, following interaction with the bacterial outer membrane proteins OmpC and OmpF. SLAM recruited a complex containing the intracellular class III phosphatidylinositol kinase Vps34, its regulatory protein kinase Vps15 and the autophagy-associated molecule beclin-1 to the phagosome, which was responsible for inducing the accumulation of phosphatidylinositol-3phosphate, a regulator of both NOX2 function and phagosomal or endosomal fusion. Thus, SLAM

\section{(C) 2010 Nature America, Inc. All rights reserved.}

Correspondence should be addressed to S.B.B. (sberger@bidmc.harvard.edu) or C.T. (terhorst@ bidmc.harvard.edu).

7 These authors contributed equally to this work.

Note: Supplementary information is available on the Nature Immunology website.

\section{AUTHOR CONTRIBUTIONS}

S.B.B., X.R. and C.T. designed the experiments, analyzed the data and wrote the manuscript; S.B.B. and X.R. did all of the experiments except for the in vivo killing experiments and some of the Jurkat recognition and immunoprecipitation experiments; C.M. did many of the immunoprecipitation experiments; G.W. helped with the confocal microscopy and Jurkat recognition experiments; W.A.F. initiated the killing experiments, was involved in the oxidative burst experiments and set up the Jurkat recognition experiments; G.L. set up the immunoprecipitation; E.C. did some of the Jurkat recognition experiments; M.K. did the restriction fragment length polymorphism analysis and helped make some of the stable transfectants; L.R. did the HPLC analysis; N.W. produced the Slamf1 $1^{-1}$ mice; M.B. provided the MHC class II-eGFP mice and helped with analysis of the Slamf1-1- MHC class II-eGFP experiments; J.R.R. helped with production of the SLAM-mCherry construct; H.C.R. helped with the design, data acquisition and interpretation of the confocal microscopy; and C.T. supervised the study.

\section{COMPETING FINANCIAL INTERESTS}

The authors declare no competing financial interests.

Reprints and permissions information is available online at http://npg.nature.com/reprintsandpermissions/. 
connects the Gram-negative bacterial phagosome to ubiquitous cellular machinery responsible for the control of bacterial killing.

Diverse macrophage receptors act together to recognize bacteria via conserved structures on the bacterial surface and facilitate phagocytosis and/or signaling that initiates the innate immune response and triggers subsequent activation of adaptive immunity. These bacterial receptors include scavenger receptors, C-type lectins, integrins, Toll-like receptors (TLRs) and Siglec proteins, which recognize conserved bacterial moieties ranging from lipopolysaccharide (LPS) on Gram-negative bacteria to peptidoglycan and lipoteichoic acid on Gram-positive bacteria ${ }^{1}$. Many of these receptors are somewhat promiscuous, recognizing multiple ligands with varying degrees of specificity and affinity ${ }^{2}$. Whether and how these macrophage cell surface receptors, some of which enter the phagosome, control key steps in microbicidal functions, such as the production of reactive oxygen species or phagolysosomal fusion, is not well understood at present.

Receptors of the signaling lymphocyte-activation molecule family (SLAMF), encoded by Slamf1-Slamf9 in the mouse, are adhesion molecules on the surface of most hematopoietic cells that serve as costimulatory molecules that initiate distinct signal-transduction networks in T cells, natural killer cells and antigen-presenting cells ${ }^{3,4}$. Both functional and structural studies have demonstrated that the ectodomains of the SLAMF receptors are homophilic or self ligand receptors, except SLAMF2 (CD48), which uses both SLAMF4 (CD244) and $\mathrm{CD} 2$ as its counter-ligands. These receptors not only operate as costimulatory molecules in the adaptive immune system but also participate in lineage-commitment steps of hematopoiesis and natural killer T cell development, as well as in the functional regulation of natural killer cells, neutrophils, dendritic cells, macrophages and platelets ${ }^{3}$. In addition, SLAM (SLAMF1; CD150) is a receptor for measles virus ${ }^{5}$.

As most SLAMF receptors are expressed on the surface of myeloid cells, we decided to examine the role of SLAMF receptors in innate immune responses other than natural killer cell functions. This idea was supported by several studies of the role of SLAM receptors in responses to bacteria or bacterial components ${ }^{6,7}$. In this report we show that SLAM (SLAMF1) had a role in innate immune responses to inoculation with Escherichia coli or an attenuated Salmonella typhimurium strain negative for production of the Salmonella pathogenicity island 2 type III secretion system $\mathrm{SseB}$ protein $\left(\mathrm{SseB}^{-}\right)$by regulating bacteriocidal activity in the phagosome of macrophages. After interaction with E. coli, SLAM entered the bacterial phagosome, where it mobilized a ubiquitous enzyme complex involved in organelle fusion through the production of phosphatidylinositol-3-phosphate $(\text { PtdIns(3)P })^{8}$. Because PtdIns(3)P also regulates the activity of the NADPH oxidase (NOX2) complex in phagosomes, SLAM-deficient macrophages killed inefficiently. Thus, SLAM is not only a self ligand and a receptor for measles virus but also a bacterial sensor that, like some TLRs, regulates intracellular enzyme activities involved in the removal of Gram-negative bacteria.

\section{RESULTS}

\section{Inefficient killing by SLAM-deficient macrophages}

As SLAM is expressed on the surface of macrophages and because SLAM is involved in the regulation of cytokine secretion by human and mouse macrophages and dendritic cells ${ }^{3,7}$, we set out to evaluate a possible role for SLAM in innate immune responses. We therefore inoculated mice with double knockout of SLAM and recombination-activating gene 1 (Slamf1-/- $\operatorname{Rag} 1^{-1-}$ mice) with a 1:1 mixture of the attenuated $S$. typhimurium $\mathrm{SseB}^{-} \operatorname{strain}^{9}$ and virulent wild-type $S$. typhimurium strain 14028s. At $48 \mathrm{~h}$ after infection, the double- 
knockout mice cleared neither bacteria, as judged by strain-selective bacterial counts in the spleen (Fig. 1a). In contrast, $\mathrm{Slamf}^{\mathrm{I}^{++}} \mathrm{Rag}^{\mathrm{I}^{-/-}}$mice cleared the attenuated strain but not the wild-type bacteria (Fig. 1a). This weakened innate immune response of the double-knockout mice was reflected in the impaired clearing of the same attenuated $S$. typhimurium $\mathrm{SseB}^{-}$ strain by Slamf1 $^{-1-}$ mice but not SLAM-sufficient wild-type mice (Fig. 1b).

The inefficient responses of the mutant mice to bacterial inoculation were caused by defective killing of nonopsonized $S$. typhimurium $\mathrm{SseB}^{-}$by $S l a m f 1^{-1-}$ macrophages, as determined by gentamicin killing assays (Fig. 1c). Whereas killing of E. coli was also impaired (Fig. 1d), we observed no defect in the response to Gram-positive $S$. aureus (Fig. 1e). The differences in macrophage killing could not be attributed to an obvious defect in bacterial uptake because we found no difference between mutant and wild-type killing at early time points of the gentamicin assay (Fig. 1c,d). In addition, we found no difference between wild-type and mutant macrophages in a cytofluorometry-based phagocytosis assay with $S$. typhimurium $\mathrm{SseB}^{-}$or $E$. coli expressing enhanced green fluorescent protein (eGFP; Fig. 1f). We excluded the possibility of developmental defects (Supplementary Fig. 1) or an influence of the genetic background of the mutant mice (Supplementary Fig. 2) because Slamf ${ }^{+/+}$and Slamf $1^{-/-}$macrophages had no difference in their expression of cell surface markers (Supplementary Fig. 1) and because the killing of E. coli by Slamf1 ${ }^{-1-}$ C57BL/6 (B6) and Slamf1 $^{-1-}$ BALB/c macrophages was equally impaired (Supplementary Fig. 2). The latter finding was not unexpected, as both $S l a m f 1^{-/-}$BALB/c and $S l a m f 1^{-/-}$B6 mice contain the SLAMF haplotype II Slamf1-Slamf7locus ${ }^{3}$, derived from the original 129 embryonic stem cells ${ }^{7}$ (Supplementary Figs. 3 and 4). Thus, the cell surface receptor SLAM positively regulates the killing of Gram-negative bacteria by macrophages.

\section{SLAM regulates NOX2 activity in E. coli-containing phagosomes}

One of the key mechanisms by which phagocytes can kill bacteria uses the NOX2 enzyme complex, composed of the plasma membrane-bound proteins $\mathrm{p} 22^{\mathrm{phox}}$ and $\mathrm{gp} 91^{\mathrm{phox}}$ and the cytosolic proteins $\mathrm{p} 40^{\text {phox }}, \mathrm{p} 47^{\text {phox }}, \mathrm{p} 67^{\text {phox }}, \operatorname{Rac} 1$ and Rac 2 , which are recruited to the membrane complex ${ }^{10}$. In macrophages, the active NOX2 enzyme in the phagosomal membrane is responsible for the reduction of $\mathrm{O}_{2}$ to $\mathrm{O}_{2}^{-}$, which is further converted in the lumen of the phagosome to superoxide. By using the chemiluminescence detector lucigenin ${ }^{9}$ to detect the production of superoxide in the cell, we found that primary $S l a m f 1^{-/-}$ macrophages produced less reactive oxygen in response to $E$. coli than did wild-type macrophages (Fig. 2a and Supplementary Fig. 5). In contrast, NOX2 activity induced by $S$. aureus was not affected in the SLAM-deficient macrophages. Thus, SLAM is a positive regulator of NOX2 activity in macrophages. We confirmed that with the finding that in response to E. coli, NOX2 activity was greater in Slamf1-transfected RAW264.7 mouse macrophages (Supplementary Fig. 6), which themselves are SLAM deficient.

To test the hypothesis that SLAM specifically regulates NOX2 activity in the lumen of Gram-negative phagosomes, we compared the $\mathrm{pH}$ of E. coli- and $S$. aureus-containing phagosomes in freshly isolated macrophages. The fluorescence assay we used is based on the principle that conversion of the reactive oxygen species that are produced by active NOX2 to $\mathrm{HO}_{2}$ and $\mathrm{H}_{2} \mathrm{O}_{2}$ consumes protons, which enter the phagosome via a proton pump $^{11}$. Thus, impairment in the activity of the NOX2 enzyme would result in less proton consumption in the bacterial phagosome and the $\mathrm{pH}$ would be lowered more rapidly ${ }^{12}$. To test that idea, we used the $\mathrm{pH}$-sensitive dye $\mathrm{pHrodo}^{13}$, coupled to the outer surface of either $E$. coli or $S$. aureus, to monitor the decrease in $\mathrm{pH}$ in bacterial phagosomes by cytofluorometry.

Indeed, the E. coli-containing phagosomes acidified more rapidly in Slamf1 ${ }^{-1-}$ macrophages than in wild-type macrophages. In fact, acidification in the $S l a m f 1^{-1-}$ macrophage-derived 
phagosomes was similar to that of phagosomes from mice deficient in the gp91 9 phox component of NOX2 ( $\mathrm{Cybb}^{-/-}$; called ' $g p 91 \mathrm{phox}^{-/-}$here; Fig. 2b). In contrast, acidification of the $S$. aureus-containing phagosomes took place at the same rate in $S l a m f 1^{-1-}$ and wildtype macrophages, whereas the $\mathrm{pH}$ was lower in the $S$. aureus-containing phagosomes from gp91phox ${ }^{-/-}$mice (Fig. 2b). Thus, the defective function of NOX2 in Slamf $1^{-1-}$ macrophages resided in the $E$. coli-containing phagosomes.

One plausible explanation for the specificity for $E$. coli could be that the impaired NOX2 response by $S l a m f 1^{-/-}$macrophages involves an indirect response to lipopolysaccharide (LPS) due to a convergence of SLAM- and TLR4-induced signal-transduction networks. However, NOX2 activation by LPS was impaired only in TLR4-deficient macrophages, not in primary Slamf $1^{-1-}$ macrophages (Fig. 2c). Predictably, NOX2 responses by macrophages to peptidoglycan or the phorbol ester PMA were not affected by SLAM deficiency (Fig. 2c). We conclude that SLAM positively regulates the function of NOX2 in E. coli-containing phagosomes but not in $S$. aureus-containing phagosomes.

\section{Delayed maturation in the absence of SLAM}

Because lysosomal enzymes contribute to the elimination of bacteria by macrophages ${ }^{14}$, we assessed whether phagolysosomal maturation was dysregulated in the absence of SLAM. For this, we transfected primary macrophages with LAMP-1 (an established lysosomal marker) conjugated to red fluorescent protein (RFP) and initiated phagocytosis of eGFPexpressing $E$. coli ( $E$. coli-eGFP) or $S$. aureus ( $S$. aureus-eGFP). When we evaluated the staining of $E$. coli-containing phagosomes with RFP-conjugated LAMP-1 by fluorescence microscopy, we observed a quantitative delay in the recruitment of LAMP-1 in Slamf1-1macrophages relative to its recruitment in wild-type cells. In contrast, we found no difference in the recruitment of LAMP-1 to $S$. aureus-containing phagosomes in wild-type or Slamf1 $1^{-1-}$ macrophages (Fig. 3a). Thus, consistent with the impaired bacterial killing and diminished NOX2 activity, the maturation of phagosomes containing E. coli, but that of not those containing $S$. aureus, was affected by the absence of SLAM in macrophages.

We confirmed a role for SLAM in phagolysosomal maturation by analyzing phagosomes isolated by sucrose-gradient flotation ${ }^{15}$. We initiated phagocytosis by adding $3-\mu \mathrm{m}$ polystyrene beads coated with a crude preparation of $E$. coli outer membrane extracts to mock-transfeted or Slamf1-transfected RAW264.7 macrophages. At various times (30, 60 or $120 \mathrm{~min}$ ) after the initiation of phagocytosis, we isolated the organelles on the basis of bead buoyancy. Phagosomes purified from SLAM ${ }^{+}$macrophages had more LAMP-1 at 60 and 120 min after the initiation of phagocytosis than did those isolated from mock- transfected macrophages (Fig. 3b).

In the next set of experiments we used a second phagolysosomal marker: eGFP-tagged major histocompatibility complex (MHC) class II. We compared phagosomal maturation in macrophages from a reporter mouse in which the gene encoding MHC class II is replaced with a version that encodes MHC class II tagged with eGFP (MHC class II-eGFP) ${ }^{16}$ with that of the progeny of MHC class II-eGFP mice crossed with Slamf1 $^{-1-}$ mice (Slamf1-1MHC class II-eGFP mice). We allowed primary macrophages to phagocytose $3-\mu \mathrm{m}$ polystyrene beads coated with a crude preparation of $E$. coli outer membrane extracts and monitored translocation to phagolysosomes by confocal microscopy. We detected considerably fewer MHC class II-positive phagolysosomes in Slamf $^{-/-}$MHC class II-GFP macrophages than in wild-type MHC class II-GFP macrophages $60 \mathrm{~min}$ after initiation of phagocytosis (Fig. 3c). However, we observed no difference $120 \mathrm{~min}$ after the initiation of phagocytosis (data not shown). This confirmed that trafficking of cargo to the phagolysosome was delayed in the absence of SLAM. 
As an alternative approach to determining the arrival of cargo in the phagolysosome, we loaded lysosomes with Texas red-dextran before initiating phagocytosis with coated $3-\mu \mathrm{m}$ polystyrene beads. We quantified colocalization of the beads and Texas red at various time points by fluorescence microscopy. Whereas Slamf $^{+/+}$macrophages contained coated beads in the dextran-loaded lysosomes $60 \mathrm{~min}$ after the initiation of phagocytosis, we detected only a small number of beads in Slamf1 $^{-1-}$ lysosomes (Fig. 3d).

The consequence of the absence of SLAM was already detectable at an earlier stage of phagosome maturation, as recruitment of the tethering molecule EEA1 and the small GTPase Rab5.GTP ${ }^{17,18}$ was delayed, as judged by quantitative fluorescence microscopy (Fig. 4). The specificity of the role of SLAM in bacterial phagosomal maturation was further emphasized by the finding that SLAM deficiency had no effect on the formation of transferrin-loaded recycling endosomes or the formation of low-density lipoproteinendolysosomes, which are dependent on fusion events involving EEA1 (Supplementary Figs. 7 and 8). We observed the delay in phagosomal maturation, as judged by Rab5 localization, in primary macrophages from both Slamf1 $^{-1-}$ BALB/c mice and Slamf1 ${ }^{-1-}$ B6 mice (Supplementary Fig. 9), and this defect was not caused by the impaired NOX2 function itself, because gp91 phox $_{\text {-deficient and }} 440^{\text {phox }}$-deficient macrophages had a normal phagolysosomal maturation (data not shown). Together, the outcomes of these experimental approaches demonstrate that both NOX2 activity in the E. coli-containing phagosome and the progression of phagolysomal maturation are positively controlled by the cell surface receptor SLAM.

\section{Entry of SLAM into E. coli-containing phagosomes}

Because of its effect on two key microbicidal phagosomal processes, we reasoned that any SLAM-dependent mechanism(s) would require entry of SLAM into the Gram-negative phagosome. To test our hypothesis, we first transfected SLAM-deficient RAW264.7 macrophages with cDNA encoding a fusion protein of SLAM and the red fluorescent protein mCherry (SLAM-mCherry) before phagocytosis of $E$. coli-eGFP or $S$. aureus-eGFP. Quantitative fluorescence microscopy demonstrated that SLAM-mCherry localized together with E. coli-eGFP but not with $S$. aureus-eGFP in transfectant cells (Fig. 5a). Furthermore, we also detected SLAM in phagosomes isolated from SLAM-transfected RAW264.7 macrophages (Fig. 5b). These observations demonstrate that SLAM enters the phagosome with the same degree of specificity as its regulation of microbicidal activity (Figs. 2 and 3).

\section{SLAM recognizes bacterial OmpC and/or OmpF}

Because the deletion of SLAM affected E. coli-related phagosomal events, we reasoned that SLAM recognizes a surface component of the bacterium. To test our hypothesis, we developed a sensitive signal-amplification assay in which we transfected a fusion protein of the mouse SLAM ectodomain and the intracellular region of human CD3 $\zeta$ into Jurkat human $\mathrm{T}$ cells together with a luciferase reporter driven by the promoter of the gene encoding interleukin 2. With this cell-surface based assay we determined that SLAM recognized both $E$. coli and $S$. typhimurium $\mathrm{SseB}^{-}$(Fig. 6a). In contrast, $S$. aureus did not induce a response above the background response caused by homophilic SLAM-SLAM interactions. We confirmed the specificity of the direct interaction of SLAM with E. coli by two experiments: first, recognition of $E$. coli was abolished after removal of the N-terminal ecto-domain (immunoglobulin V) of SLAM (Fig. 6b and Supplementary Fig. 10); and second, two monoclonal antibodies directed against SLAM ${ }^{19}$ blocked the response to $E$. coli (Fig. 6c and data not shown). Recognition of these Gram-negative bacteria was independent of LPS binding because Jurkat cells do not express TLR4. Furthermore, in response to $E$. coli, TLR4-deficient macrophages are not impaired in phagocytosis ${ }^{20}$ or NOX2 function ${ }^{9}$. 
Additionally, SLAM does not directly bind to LPS because NOX2 activation by LPS was identical in primary macrophages from wild-type and Slamf1 ${ }^{-1-}$ mice (Fig. 2c).

To determine which component of the $E$. coli outer membrane might be involved in recognition by SLAM, we analyzed the crude $E$. coli membrane extract used for coating the polystyrene beads in the phagocytosis assays (Figs. 3-5). Nonquantitative mass spectrometry-based analyses indicated that the outer membrane porins were principal components of the preparation used (data not shown). We therefore determined whether SLAM recognized $E$. coli mutants lacking one or more of the $E$. coli outer membrane porins. We found that an E. coli mutant (HN705) lacking both of the outer membrane porins OmpC and $\mathrm{OmpF}$ was not recognized by SLAM, whereas E. coli mutants lacking either OmpC or OmpF were partially recognized (Fig. 6d). In contrast, many other E. coli variants or mutants were recognized by SLAM (Supplementary Fig. 11). The most plausible explanation for these observations is that the membrane-distal SLAM ectodomain binds to one or more of the extracellular loops of OmpC and OmpF, which have similar sequences ${ }^{21}$ (Supplementary Fig. 12).

To evaluate the role of OmpC in a SLAM-dependent physiological process, we used purified OmpC to induce NOX2 activity in SLAM-deficient and SLAM-sufficient primary macrophages (Fig. 2c). Indeed, SLAM deficiency resulted in a lower NOX2 response to the purified protein, which indicated a relationship between SLAM function and this bacterial component. In contrast, NOX2 responses in macrophages to LPS, peptidoglycan, CpG (a TLR9 ligand) or PMA were not affected by the alteration of SLAM (Fig. 2c and data not shown). Additionally, we made use of the HN705 E. coli mutant doubly deficient in OmpC and OmpF to functionally confirm that these proteins were the targets of the SLAM receptor (Supplementary Fig. 13). As expected, SLAM-deficient macrophages had less NOX2 activity in response to wild-type E. coli (JM101). However, we observed no defect in response to the HN705 double mutant. Together these data demonstrate that SLAM itself is able to sense the molecular signature of Gram-negative bacteria by recognizing OmpC and most probably OmpF and as a consequence enters the phagosome, where it directs NOX2 activity and phagosomal maturation.

\section{SLAM regulates Ptdlns(3)P production in phagosomes}

A potential mechanism by which SLAM could control two seemingly disparate microbicidal functions of macrophages after uptake of E. coli (NOX2 activity and phagosomal maturation) involves PtdIns(3)P. First, PtdIns(3)P, located in the outer layer of the phagosomal lipid bilayer ${ }^{22,23}$, binds to the PX domain of the NOX2 subunit $\mathrm{p} 40^{\mathrm{phox}}$ (NCF4), which is required for both assembly and stabilization of the NOX2 enzyme ${ }^{24}$. Second, PtdIns(3)P in the outer leaflet of the phagosome and endosome lipid bilayer interacts with the FYVE domain of the tethering molecule EEA1, a requisite step for successful phagosomal and endosomal maturation ${ }^{25}$.

To test the idea that absence of SLAM affects the amount of PtdIns(3)P in the outer leaflet of the phagosomal lipid bilayer, we transfected cDNA encoding a PtdIns(3)P-binding reporter into wild-type and SLAM-deficient primary macrophages. After initiating phagocytosis with $E$. coli expressing the red fluorescent protein DsRed, we monitored the amount of PtdIns(3)P in E. coli-containing phagosomes by live-cell spinning-disc confocal microscopy. The production of PtdIns(3)P in the E. coli-containing phagosomes of $S_{\text {Slamf1 }}{ }^{-1-}$ macrophages was defective (Fig. 7a). We independently confirmed that result by quantitative fluorescence microscopy of the phagocytosis of $3-\mu \mathrm{m}$ beads coated with an $E$. coli outer membrane extract. Whereas in wild-type peritoneal macrophages PtdIns(3)P peaked in phagosomes at $15 \mathrm{~min}$ after the initiation of phagocytosis (Fig. 7b), it reached its peak at 45 min after the initiation of phagocytosis in SLAM-deficient macrophages (Fig. 
$7 \mathrm{~b})$. We obtained the same result when we examined bone marrow-derived macrophages (data not shown). We did not detect any PtdIns(3)P by this analysis in the plasma membrane when we used $15-\mu \mathrm{m}$ coated polystyrene beads, which cannot be phagocytosed (data not shown). Furthermore, the delay in appearance of PtdIns(3)P in the phagosomal lipid bilayer coincided with the delay in recruitment of the tethering EEA1 dimer (Fig. 4a), which depends on the binding of PtdIns(3)P to its FYVE domain ${ }^{26}$.

In a biochemical experiment, we labeled primary macrophages with tritiated myoinositol, the PtdIns(3)P precursor. After the initiation of phagocytosis, we detected less PtdIns(3)P in SLAM-deficient macrophages than in wild-type macrophages, as determined by highpressure liquid chromatography (HPLC) analysis (Fig. 7c). In this experiment we measured new production of PtdIns(3)P, which represents enzyme activity in the phagosomes. The outcome of the lipid analysis therefore confirmed the delay in PtdIns(3)P production in Slamf $^{-1-}$ phagosomes determined by the two fluorescence microscopy studies.

Because the experiments reported above indicated that after entering the phagosome, SLAM must be a positive regulator of PtdIns(3)P production, we next used SLAM ${ }^{+}$RAW264.7 macrophage transfectants. Quantitative fluorescence microscopy showed SLAM-enhanced mobilization of PtdIns(3)P to the phagosomes (Fig. 7d). We conclude from these experiments that in phagosomes, SLAM governs the amount of PtdIns(3)P in the membrane, which binds to $40^{\text {phox }}$ to affect the function of NOX2, and to EEA1, which influences phagosome maturation.

\section{SLAM interacts with the Vps34-Vps15-beclin-1 complex}

The most likely enzyme to regulate PtdIns(3)P in the phagosome would be the class III phosphatidylinositol kinase Vps34, which resides exclusively in intracellular membrane compartments and is the sole kinase able to convert phosphatidylinositol into $\operatorname{PtdIns}(3) \mathrm{P}^{8,27}$. Vps34, along with its regulatory protein kinase Vps15, is a critical regulator of endocytic sorting in yeast and mammalian cells ${ }^{8,28}$. However, Vps34 and Vps15 have been found to constitutively exist in a heterotrimeric complex along with the autophagy-associated molecule beclin-1 (Atg6), referred to as the 'beclin 1-phosphatidylinositol-3-kinase complex ${ }^{29,30}$. When this heterotrimeric complex is associated with the ultraviolet irradiation resistance-associated protein UVRAG, it generates PtdIns(3)P, which promotes the fusion of organelles ${ }^{31,32}$. We therefore set out to demonstrate that SLAM interacts with this enzyme complex in intracellular membranes.

To assess whether SLAM directly interacts with the Vps34-Vps15-beclin-1 complex, we expressed all four proteins together in HEK293 human embryonic kidney cells. Coimmunoprecipitation and immunoblot analysis demonstrated that SLAM interacted with the Vps34-Vps15-beclin-1 complex (Fig. 8a). Although detailed mutational analyses are needed, the main interaction seemed to be between SLAM and beclin-1. The observation that the SLAM-specific adaptor EAT-2A enhanced the interaction with Vps34 (Fig. 8a) raised the possibility that binding of EAT-2 to SLAM might stabilize the protein interactions. When we used a mutant form of SLAM lacking the cytoplasmic tail, we found that neither Vps34 nor any of its associated components was coprecipitated (Fig. 8b). We confirmed the interaction of SLAM with the beclin-1-containing complex in phagosomes by fluorescence microscopy of RAW264.7 macrophages transfected with SLAM and GFPtagged beclin-1 (Fig. 8c). The outcome of these experimental approaches showed that the presence of SLAM led to more PtdIns(3)P in the lipid bilayer of the phagosome because SLAM recruited the Vps34-Vps15-beclin-1 enzyme complex. Thus, SLAM regulated bactericidal activity in the Gram-negative phagosome through the recruitment of this complex and subsequent phagosomal maturation and NOX2 activity (Supplementary Fig. 14). 


\section{DISCUSSION}

Our experiments have demonstrated that in addition to being a costimulatory molecule, SLAM acts as a vital regulator in the innate immune defense against Gram-negative bacteria in macrophages by independently regulating two main bactericidal processes: phagosome maturation and the production of free radical species by the NOX2 complex. To regulate these microbicidal processes, SLAM first recognizes bacterial surface proteins embedded in the outer membrane of most Gram-negative bacteria ${ }^{33,34}$. Once SLAM engages the bacteria, it is actively dragged into the developing phagosome, where it is responsible for recruiting a complex containing Vps34, Vps15 and beclin-1 to the early phagosome. The recruitment of active Vps34 catalyzes the conversion of phosphatidylinositol phosphate to PtdIns(3)P, a key regulator of phagosomal maturation through the recruitment of EEA-1 via its FYVE domain and the production of reactive oxygen species by its recruitment of $\mathrm{p} 40^{\text {phox }}$ via its PX domain. This pathway, when intact, leads to the optimal and efficient elimination of Gram-negative species.

Bacteria are recognized by low-specificity, high-affinity receptors such as integrins, lectins and scavenger receptors that initiate the formation of the phagocytic synapse and intracellular signaling events. Our data have shown that SLAM is a bacterial receptor that recognizes the outer membrane proteins $\mathrm{OmpC}$ and $\mathrm{OmpF}$. OmpC has become an area of interest in the study of inflammatory bowel disease because a subset of patients with Crohn's disease have relatively high concentrations of antibodies to $\mathrm{OmpC}^{35}$. OmpC and $\mathrm{OmpF}$ are highly homologous porins that are regulated in an extremely complex way that involves a variety of growth conditions such as temperature, $\mathrm{pH}$ and osmolarity ${ }^{33}$. They fulfill many tasks that are crucial to bacterial homeostasis, including solute-protein translocation and structural integrity, and they are also thought to contribute to bacterial virulence. So far, no other receptors for OmpC or $\mathrm{OmpF}$ have been identified, to our knowledge, and only OmpA from Klebsiella pneumoniae has been described as binding to the scavenger receptors LOX-1 and SREC-I, which results in the activation of macrophages and dendritic cells in a TLR2-dependent way. The mechanism by which the interactions of SLAM with OmpC and OmpF is orchestrated is as yet undetermined.

The Vps34-Vps15-beclin-1 complex has become the focus of much work in terms of its role in the macroautophagy (autophagy) process. Autophagy is a universal process by which a cell acts to degrade superfluous materials in the cytoplasm and organelles and reutilize them as vital elements of cell survival in response to cellular stress ${ }^{36}$. Additionally, in some cases, this process is also important in immune defense against pathogens ${ }^{37}$. This phenomenon is achieved through the formation of a double-membraned structure called the autophagosome that eventually fuses with the lysosome, a process similar to phagosomal maturation. Studies have shown that the Vps34-Vps15-beclin-1 complex also associates with UVRAG and that this complex, called the autophagy complex, is indispensable to the initiation and continuance of autophagy ${ }^{31,32,36}$.

Thus, SLAM may support phagosome maturation, which itself is dependent on vesicular fusion events ${ }^{38}$, by borrowing from the ubiquitous autophagy machinery. There is also overlap between the elements used in phagosome maturation and autophagy, as TLRdependent triggering of phagocytosis recruits the autophagy proteins beclin-1 and LC 3 to the phagosome ${ }^{39}$. Additionally, optimal activation of reactive oxygen species by the NOX2 complex is essential for the initiation of autophagy ${ }^{40}$, which makes SLAM a potential candidate for its induction. Whether SLAM is also a regulator of the autophagy process in the context of stress or immunity is an area that requires further investigation. 
We conclude that after phagocytosis of Gram-negative bacteria, SLAM connects the phagosome to an enzyme system that is present in every cell; thus, we have identified a previously unknown innate receptor function that tailors the immune response to Gramnegative bacteria. Because we found that SLAMF6 (Ly 108) also recognized E. coli (data not shown) but not $S$. aureus, and because SLAMF2 is one of the receptors for FimH, a lectin on the pili of some Enterobacteriaceae, we propose that the SLAMF receptors are another family of microbial sensors. Like other innate immune receptors, such as TLRs ${ }^{41}$, SLAMF receptors are promiscuous in that they can recognize many ligands; for example, SLAMF1 reacts with self proteins, measles virus proteins and bacterial proteins.

\section{METHODS}

Methods and any associated references are available in the online version of the paper at http://www.nature.com/natureimmunology/.

\section{Supplementary Material}

Refer to Web version on PubMed Central for supplementary material.

\section{Acknowledgments}

We thank H. Remold, P. Klebba and members of the Terhorst lab for critical review of the manuscript; S. Laroux for help with initial oxidative burst experiments; A. Nohturft, M. Yaffe, P. Klebba and G. Pier for discussions; B. McCormick (University of Massachusetts) for E. coli F18; C. Nagler (University of Chicago) and P. Klemm (Technical University of Denmark) for eGFP-expressing bacteria; H. Nikaido (University of California Berkeley), P. Klebba (University of Oklahoma) and G. Pier (Harvard Medical School) for mutant strains of E. coli and $S$. aureus; M. Yaffe (Massachusetts Institute of Technology) for the p40-eGFP construct; L. Cantley (Harvard Medical School) for Vps34-Myc; R. Tsien (University of California San Diego) for the mCherry construct; J. Backer (Albert Einstein College of Medicine) for the Vps34-Vps15-V5 construct; P. Hawkins (Babraham Institute) for p40phox deficient mice; and S. Targan (Cedars-Sinai) for the highly purified OmpC preparation. Supported by the US National Institutes of Health (AI-15066 to C.T., and DK-068181 and DK-003506-20 to H.C.R.) and the Crohn's and Colitis Foundation of America (S.B. and X.R.).

\section{References}

1. Stuart LM, Ezekowitz RA. Phagocytosis and comparative innate immunity: learning on the fly. Nat Rev Immunol. 2008; 8:131-141. [PubMed: 18219310]

2. Aderem A, Ulevitch RJ. Toll-like receptors in the induction of the innate immune response. Nature. 2000; 406:782-787. [PubMed: 10963608]

3. Calpe $S$, et al. The SLAM and SAP gene families control innate and adaptive immune responses. Adv Immunol. 2008; 97:177-250. [PubMed: 18501771]

4. Rethi B, et al. SLAM/SLAM interactions inhibit CD40-induced production of inflammatory cytokines in monocyte-derived dendritic cells. Blood. 2006; 107:2821-2829. [PubMed: 16317102]

5. Tatsuo H, Ono N, Tanaka K, Yanagi Y. SLAM (CDw150) is a cellular receptor for measles virus. Nature. 2000; 406:893-897. [PubMed: 10972291]

6. Malaviya R, Gao Z, Thankavel K, van der Merwe PA, Abraham SN. The mast cell tumor necrosis factor alpha response to FimH-expressing Escherichia coli is mediated by the glycosylphosphatidylinositol-anchored molecule CD48. Proc Natl Acad Sci USA. 1999; 96:81108115. [PubMed: 10393956]

7. Wang N, et al. The cell surface receptor SLAM controls T cell and macrophage functions. J Exp Med. 2004; 199:1255-1264. [PubMed: 15123745]

8. Backer JM. The regulation and function of class III PI3Ks: novel roles for Vps34. Biochem J. 2008; 410:1-17. [PubMed: 18215151]

9. Laroux FS, Romero X, Wetzler L, Engel P, Terhorst C. Cutting edge: MyD88 controls phagocyte NADPH oxidase function and killing of gram-negative bacteria. J Immunol. 2005; 175:5596-5600. [PubMed: 16237045] 
10. Lambeth JD. NOX enzymes and the biology of reactive oxygen. Nat Rev Immunol. 2004; 4:181189. [PubMed: 15039755]

11. Segal AW. How neutrophils kill microbes. Annu Rev Immunol. 2005; 23:197-223. [PubMed: 15771570]

12. Savina A, et al. NOX2 controls phagosomal $\mathrm{pH}$ to regulate antigen processing during crosspresentation by dendritic cells. Cell. 2006; 126:205-218. [PubMed: 16839887]

13. Miksa M, Komura H, Wu R, Shah KG, Wang P. A novel method to determine the engulfment of apoptotic cells by macrophages using pHrodo succinimidyl ester. J Immunol Methods. 2009; 342:71-77. [PubMed: 19135446]

14. Blander JM, Medzhitov R. On regulation of phagosome maturation and antigen presentation. Nat Immunol. 2006; 7:1029-1035. [PubMed: 16985500]

15. Trivedi V, Zhang SC, Stockinger W, Nohturfft AA. Cell-free scintillation proximity assay for studies on lysosome-to-phagosome targeting. Sci STKE. 2007; 2007:13.

16. Boes M, et al. T-cell engagement of dendritic cells rapidly rearranges MHC class II transport. Nature. 2002; 418:983-988. [PubMed: 12198548]

17. Kitano M, Nakaya M, Nakamura T, Nagata S, Matsuda M. Imaging of Rab5 activity identifies essential regulators for phagosome maturation. Nature. 2008; 453:241-245. [PubMed: 18385674]

18. Rohde K, Yates RM, Purdy GE, Russell DG. Mycobacterium tuberculosis and the environment within the phagosome. Immunol Rev. 2007; 219:37-54. [PubMed: 17850480]

19. Howie D, et al. Molecular dissection of the signaling and costimulatory functions of CD150 (SLAM): CD150/SAP binding and CD150-mediated costimulation. Blood. 2002; 99:957-965. [PubMed: 11806999]

20. Yates RM, Russell DG. Phagosome maturation proceeds independently of stimulation of toll-like receptors 2 and 4. Immunity. 2005; 23:409-417. [PubMed: 16226506]

21. Basle A, Rummel G, Storici P, Rosenbusch JP, Schirmer T. Crystal structure of osmoporin OmpC from E. coli at 2.0 A. J Mol Biol. 2006; 362:933-942. [PubMed: 16949612]

22. Yeung T, Ozdamar B, Paroutis P, Grinstein S. Lipid metabolism and dynamics during phagocytosis. Curr Opin Cell Biol. 2006; 18:429-437. [PubMed: 16781133]

23. Vieira OV, et al. Distinct roles of class I and class III phosphatidylinositol 3-kinases in phagosome formation and maturation. J Cell Biol. 2001; 155:19-26. [PubMed: 11581283]

24. Bissonnette SA, et al. Phosphatidylinositol 3-phosphate-dependent and -independent functions of $\mathrm{p} 40^{\text {phox }}$ in activation of the neutrophil NADPH oxidase. J Biol Chem. 2008; 283:2108-2119. [PubMed: 18029359]

25. Lawe DC, et al. Sequential roles for phosphatidylinositol 3-phosphate and Rab5 in tethering and fusion of early endosomes via their interaction with EEA1. J Biol Chem. 2002; 277:8611-8617. [PubMed: 11602609]

26. Psachoulia E, Sansom MSP. PX- and FYVE-mediated interactions with membranes: simulation studies. Biochemistry. 2009; 48:5090-5095. [PubMed: 19408958]

27. Yan Y, Backer JM. Regulation of class III (Vps34) PI3Ks. Biochem Soc Trans. 2007; 35:239-241. [PubMed: 17371248]

28. Kinchen JM, et al. A pathway for phagosome maturation during engulfment of apoptotic cells. Nat Cell Biol. 2008; 10:556-566. [PubMed: 18425118]

29. Juhasz G, et al. The class III PI(3)K Vps34 promotes autophagy and endocytosis but not TOR signaling in Drosophila. J Cell Biol. 2008; 181:655-666. [PubMed: 18474623]

30. Lindmo K, et al. The PI 3-kinase regulator Vps15 is required for autophagic clearance of protein aggregates. Autophagy. 2008; 4:500-506. [PubMed: 18326940]

31. Matsunaga K, et al. Two Beclin 1-binding proteins, Atg14L and Rubicon, reciprocally regulate autophagy at different stages. Nat Cell Biol. 2009; 11:385-396. [PubMed: 19270696]

32. Zhong Y, et al. Distinct regulation of autophagic activity by Atg14L and Rubicon associated with Beclin 1-phosphatidylinositol-3-kinase complex. Nat Cell Biol. 2009; 11:468-476. [PubMed: 19270693]

33. Basle A, Rummel G, Storici P, Rosenbusch JP, Schirmer T. Crystal structure of osmoporin OmpC from E. coli at 2.0 A. J Mol Biol. 2006; 362:933-942. [PubMed: 16949612] 
34. Yoshida T, Qin L, Egger LA, Inouye M. Transcription regulation of ompF and ompC by a single transcription factor, OmpR. J Biol Chem. 2006; 281:17114-17123. [PubMed: 16618701]

35. Landers CJ, et al. Selected loss of tolerance evidenced by Crohn's disease-associated immune responses to auto- and microbial antigens. Gastroenterology. 2002; 123:689-699. [PubMed: 12198693]

36. Glick D, Barth S, Macleod KF. Autophagy: cellular and molecular mechanisms. J Pathol. 2010; 221:3-12. [PubMed: 20225336]

37. Virgin HW, Levine B. Autophagy genes in immunity. Nat Immunol. 2009; 10:461-470. [PubMed: 19381141]

38. Desjardins M, Nzala NN, Corsini R, Rondeau C. Maturation of phagosomes is accompanied by changes in their fusion properties and size-selective acquisition of solute materials from endosomes. J Cell Sci. 1997; 110:2303-2314. [PubMed: 9378779]

39. Sanjuan MA, et al. Toll-like receptor signalling in macrophages links the autophagy pathway to phagocytosis. Nature. 2007; 450:1253-1257. [PubMed: 18097414]

40. Huang J, et al. Activation of antibacterial autophagy by NADPH oxidases. Proc Natl Acad Sci USA. 2009; 106:6226-6231. [PubMed: 19339495]

41. Beutler B. Microbe sensing, positive feedback loops, and the pathogenesis of inflammatory diseases. Annu Rev Immunol. 2009; 227:248-263. 
a

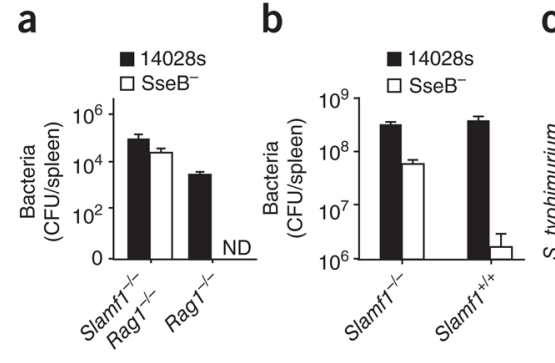

C

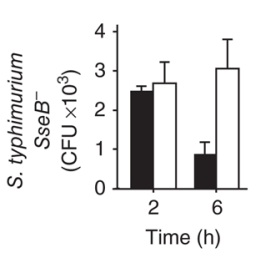

d

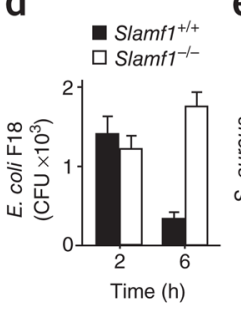

e

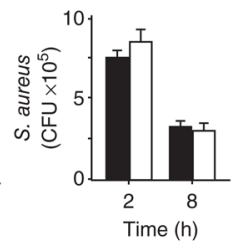

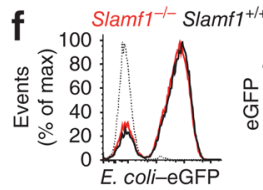

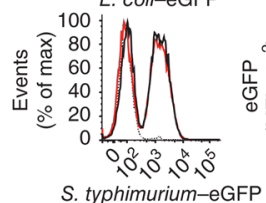

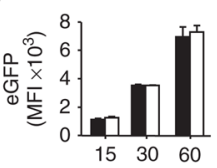

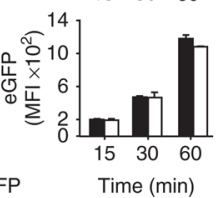

\section{Figure 1.}

SLAM controls in vivo and in vitro killing of Gram-negative bacteria by mouse macrophages. (a,b) Bacteria in the spleens of Slamf1 $^{-I_{-}}$Rag $^{-I^{-}}$and Rag $^{-I^{-}}$BALB/c mice (a) or Slamf $^{-1-}$ and Slamf $^{+/+}$BALB/c mice (b) $48 \mathrm{~h}$ after intraperitoneal injection of virulent $S$. typhimurium 14028 s or attenuated $S$. typhimurium $\mathrm{SseB}^{-}$. CFU, colony-forming units; ND, not detectable. Data are representative of four independent experiments (mean and s.d.). (c-e) Killing of bacteria by peritoneal macrophages from SlamfI $^{+/+}$and Slamf1 $^{-1-}$ BALB/c mice exposed to $S$. typhimurium $\mathrm{SseB}^{-}(\mathbf{c})$, E. coli $\mathrm{F} 18$ (d) or $S$. aureus (e), assessed by gentamycin assay. Data are representative of five independent experiments (mean and s.d.). (f) Uptake of bacteria by $\mathrm{Slamfl}^{+/+}$(red solid lines) or Slamf1 ${ }^{-1-}$ (black solid lines) BALB/c peritoneal macrophages incubated at $37^{\circ} \mathrm{C}$ with $E$. coli-eGFP or $S$. typhimurium-eGFP or by $S l a m f 1^{+/+}$macrophages incubated for $60 \mathrm{~min}$ at $4{ }^{\circ} \mathrm{C}$ with the bacteria (dotted lines). Right, mean fluorescence intensity (MFI). Data are representative of three independent experiments (mean and s.d.). 

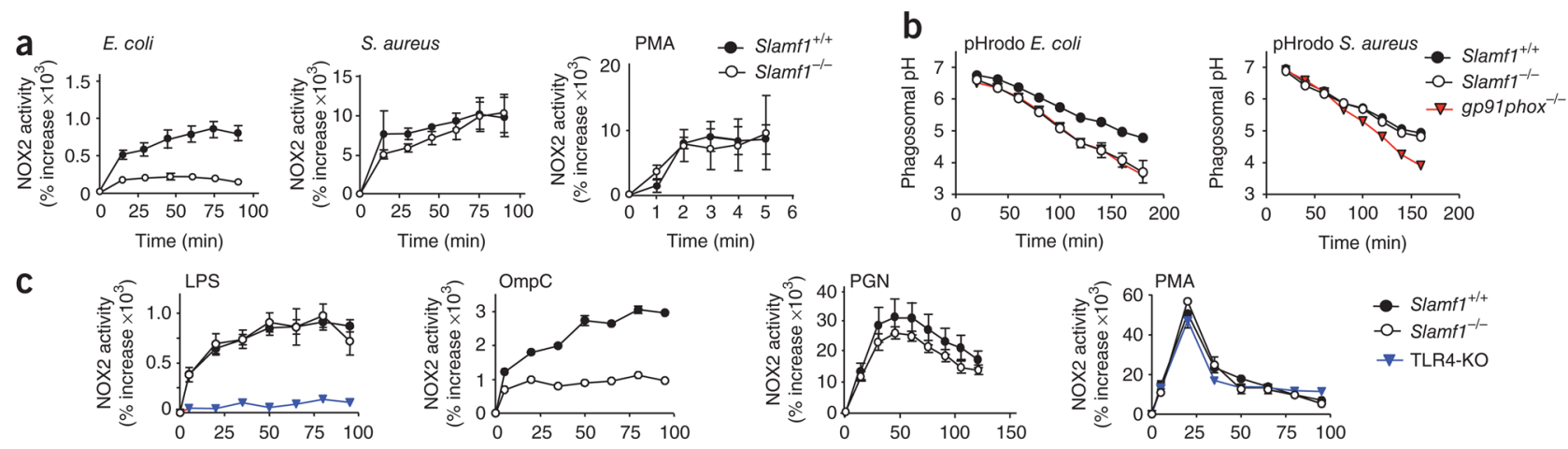

- Slamf $1^{+/+}$

-O- Slamf1

$\rightarrow$ TLR4-KO

Figure 2.

Defective NOX2 activity in primary macrophages derived from SLAM-deficient mice. (a) NOX2 activity in Slamf $1^{+/+}$and Slamf $1^{-1-}$ BALB/c peritoneal macrophages stimulated for 0-100 min with E. coli F18, S. aureus or PMA, assessed with lucigenin. Data are representative of five independent experiments (mean \pm s.d.). (b) Phagosomal $\mathrm{pH}$ of Slamf $^{+/+}$, Slamf1 $1^{-/-}$and $g p 91$ phox $^{-/-}$B6 primary macrophages loaded for 0-200 min with pHrodo-coated $E$. coli or $S$. aureus, analyzed by flow cytometry. Data are representative of three independent experiments (mean \pm s.d.). (c) NOX2 activity in primary macrophages in response to LPS, purified OmpC, peptidoglycan (PGN) or PMA, assessed with lucigenin. TLR4-KO, TLR4-deficient (strain del/Jtht; C3H). Data are representative of five independent experiments (mean \pm s.d.). 
a

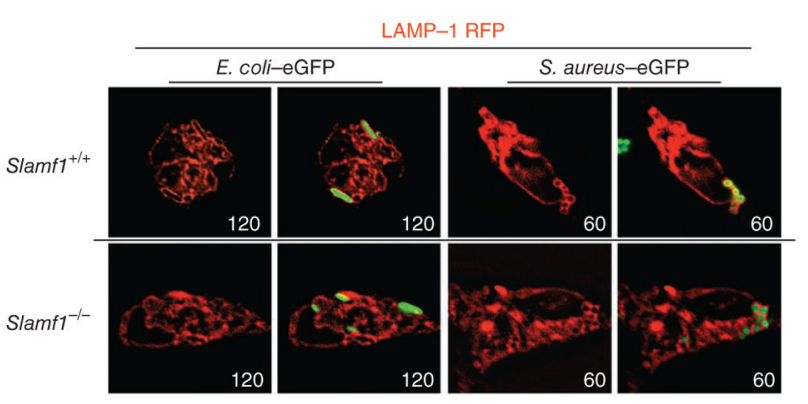

- Slamf1 ${ }^{\text {+i+ }}$

$\square$ Slamf1 $^{-/-}$

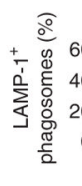

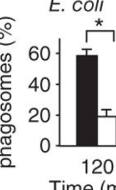

Time (min)

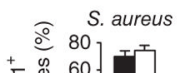

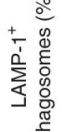

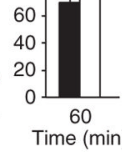

b

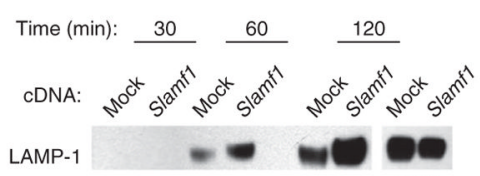

WCL
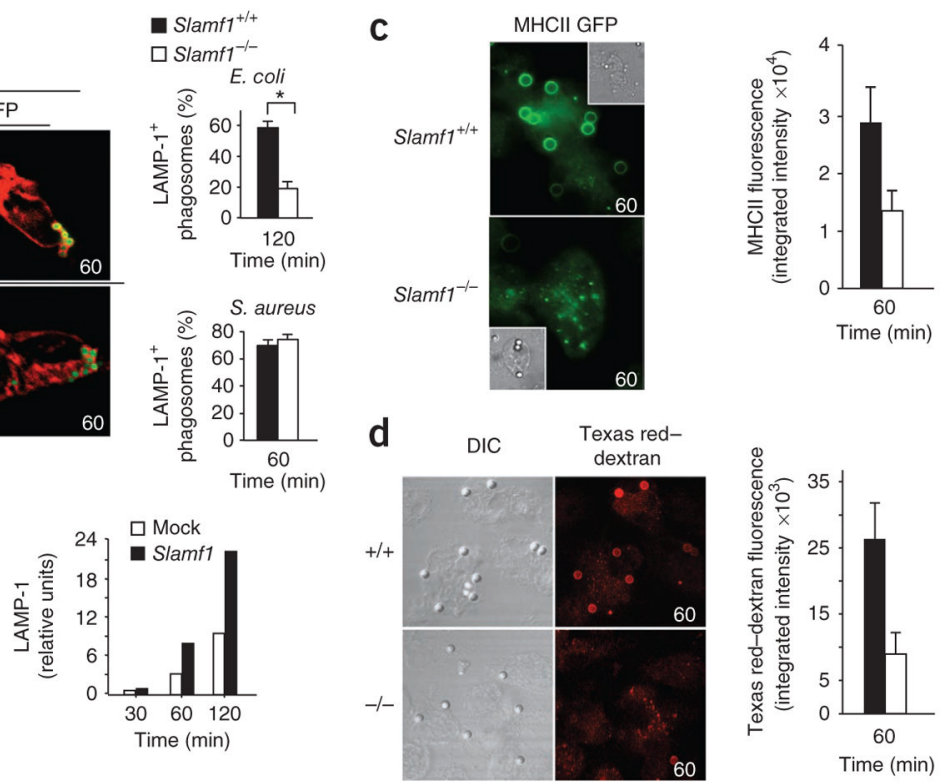

Figure 3.

Impaired phagolysosomal maturation in Slamf1 ${ }^{-1-}$ macrophages. (a) Fluorescence microscopy of $E$. coli-containing phagosomes in primary macrophages transfected with RFP-conjugated LAMP-1, showing colocalization with E. coli-eGFP or $S$. aureus-eGFP. Right, quantification of LAMP-1+ ${ }^{+}$phagosomes in the microscopy at left. (b) Immunoblot analysis of LAMP-1 in phagosomes isolated by sucrose-gradient flotation from RAW264.7 macrophages after phagocytosis by Slamf1- or mock-transfected RAW264.7 macrophages of beads coated with E. coli outer membrane extract. WCL, whole-cell lysate. Right, quantification of LAMP-1 in the immunoblot at left. (c) Localization of MHC class II-eGFP (MHCII GFP) in phagosomes of primary macrophages from Slamf $^{+/+}$or $\mathrm{Slamf1}^{-1-}$ MHC class II-eGFP (B6) mice and 3- $\mu \mathrm{m}$ beads coated with E. coli outer membrane extract. (d) Entry of 3- $\mu \mathrm{m}$ beads coated with $E$. coli outer membrane extract into lysosomes loaded with Texas red-dextran. Right (c,d), quantification of fluorescence in the microscopy at left. Numbers in bottom right corners (a,c,d) indicate time (in min). Original magnification (ac), $\times 60$. DIC, differential interference contrast. Data are representative of three combined experiments (a) or at least three independent experiments (b-d) with at least 100 beads or 80 bacteria in each (error bars, s.d.). 

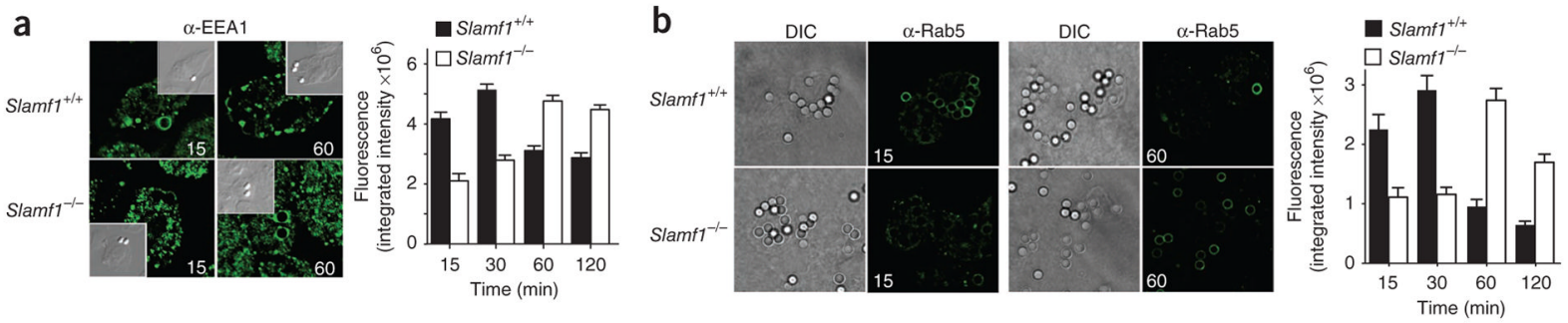

Figure 4.

Delay in early phagosomal maturation in Slamf ${ }^{-1-}$ macrophages. (a,b) Association of EEA-1 (a) or Rab5 (b) with phagosomes generated in primary macrophages by $3-\mu \mathrm{m}$ beads coated with $E$. coli outer membrane extract; numbers in bottom corners indicate time (in $\min )$. $\mathbf{a}-$, anti-. Original magnification, $\times 60$. Right $(\mathbf{a}, \mathbf{b})$, quantification of fluorescence. Data are representative of at least three independent experiments (error bars, s.d.). 
a

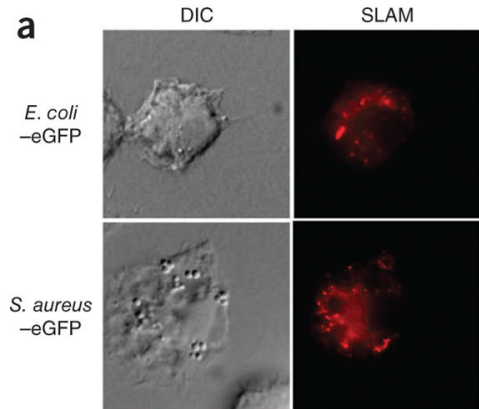

eGFP

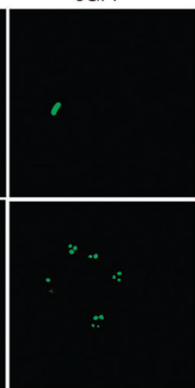

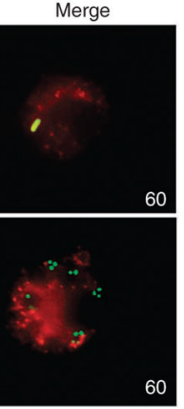

b

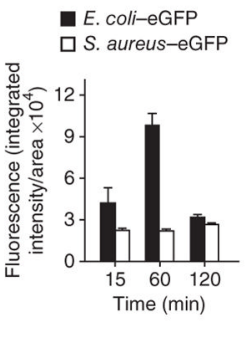

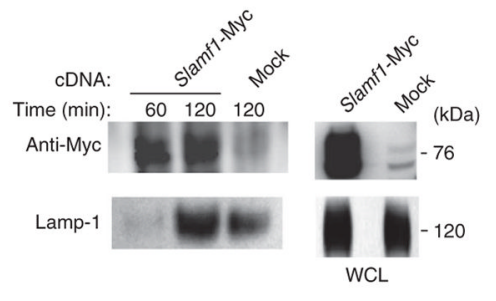

Figure 5.

SLAM enters the E. coli-containing phagosome. (a) Colocalization of SLAM and bacteria in RAW264.7 macrophages transfected with SLAM-mCherry and allowed to phagocytose E. coli-eGFP or $S$. aureus-eGFP for 0-120 min (time (in min), bottom right corners at left). Original magnification, $\times 60$. Right, quantification of fluorescence at left. Data are representative of two independent experiments with a minimum of 40 bacteria per time point (error bars, s.d.). (b) Immunoblot analysis of phagosome isolates from RAW264.7 macrophages transiently transfected with cDNA encoding Myc-tagged SLAM (Slamf1Myc) or mock transfected and allowed to phagocytose beads coated with E. coli outer membrane extract for 60 or $120 \mathrm{~min}$. $\mathrm{kDa}$, kilodaltons. Data are representative of at least three independent experiments. 

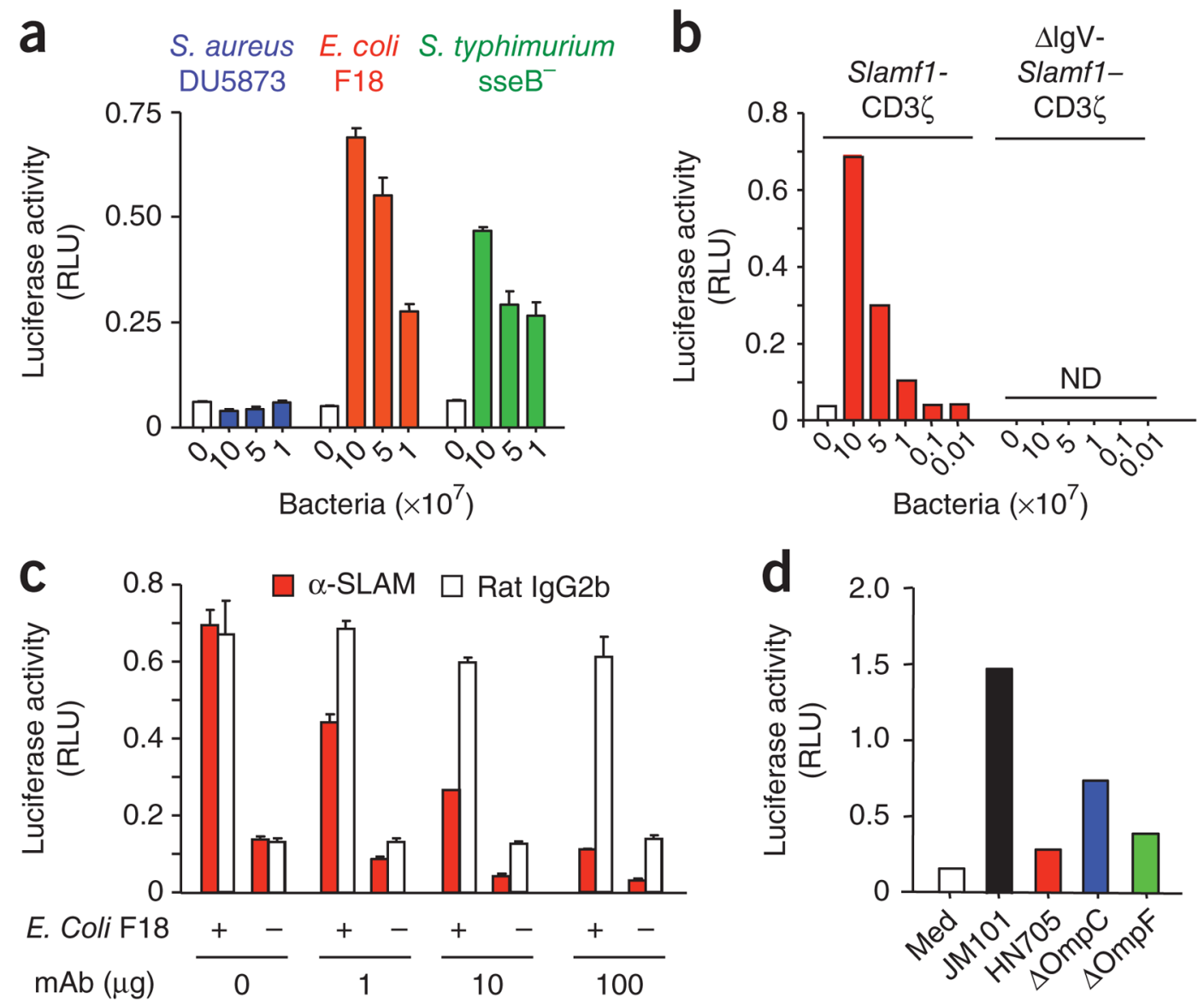

Figure 6.

SLAM recognizes $E$. coli and $S$. typhimurium $\mathrm{Sseb}^{-}$but not $S$. aureus. (a) Luciferase activity in Jurkat cells transfected with a fusion of SLAM and CD3 $\zeta$ and a luciferase reporter (as described in Results), plus a renilla luciferase reporter, then exposed to heatkilled bacteria (top). (b) Luciferase activity in Jurkat cells transfected with the fusion in a (Slamf1-CD3C) or a fusion of SLAM ectodomain construct lacking the immunoglobulin V

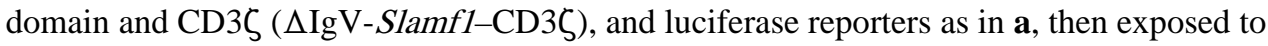
heat-killed E. coli F18. (c) Luciferase activity in Jurkat cells transfected as in a, then left uninoculated (-) or inoculated with $10 \times 10^{8}$ E. coli F18 (+), followed by the addition of monoclonal antibody (mAb; amount, under graph) 9D1 to SLAM (a-SLAM) or rat immunoglobulin G2b isotype-matched control antibody (Rat IgG2b). (d) Luciferase activity in Jurkat cells transfected as in a, then exposed to medium alone (Med), wild-type $E$. coli (JM101), or JM101 E. coli mutants lacking both Omp C and Omp F (HN705) or lacking either OmpC $(\triangle \mathrm{OmpC})$ or $\mathrm{OmpF}(\Delta \mathrm{OmpF})$. Data are representative of at least three independent experiments (error bars (a), s.d.). 

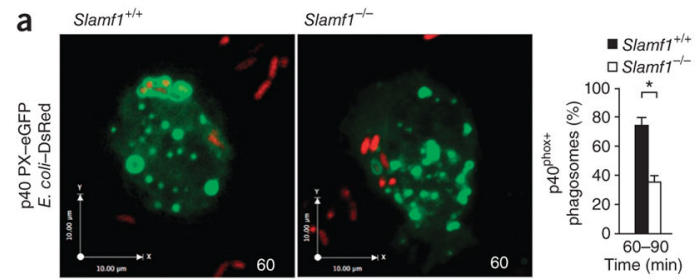
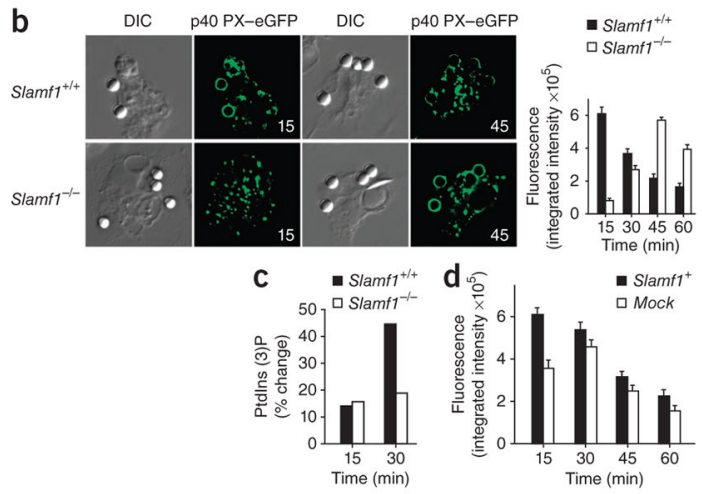

Figure 7.

PtdIns(3)P production in phagosomes of primary macrophages is controlled by SLAM. (a,b)

Production of phagosomal PtdIns(3)P in E. coli-containing phagosomes of primary peritoneal macrophages transfected with reporter cDNA encoding an eGFP-tagged PX domain of $\mathrm{p} 40^{\text {phox }}$ (p40 PX-eGFP) and treated with DsRed-expressing E. coli (E. coliDsRed; a) or beads coated with $E$. coli outer membrane extract (b). Numbers in bottom right corners indicate time (in min). Original magnification, $\times 60$. Right, quantification of fluorescence in microscopy at left. (c) HPLC analysis of the production of PtdIns(3)P in primary macrophages labeled with ${ }^{3} \mathrm{H}$-tagged myoinositol and treated with beads coated with E. coli outer membrane extract. (d) PtdIns(3)P production in RAW264.7 cells stably expressing SLAM or a mock construct, transfected with eGFP-tagged p40 PX and treated with beads coated with $E$. coli outer membrane extract. Data are from three combined experiments (a) or are representative of at least three independent experiments (b,d) or two independent experiments (c) with at least 100 beads or bacteria per experiment (error bars, s.d.). 
a

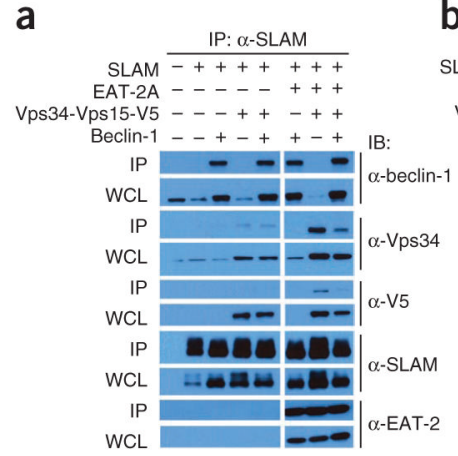

b

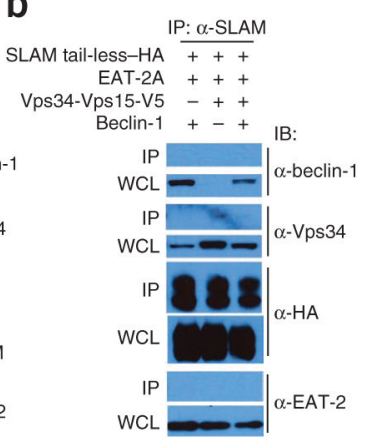

C

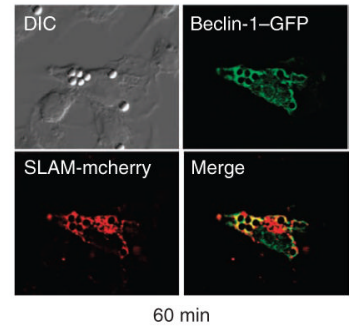

Figure 8.

SLAM recruits the intracellular Vps34-Vps15-beclin-1 complex to the phagosome. (a,b) Immunoassay of 293 cells transfected with various combinations of full-length SLAM (a) or hemagglutinin-tagged (-HA) tailless SLAM (b), EAT-2A, V5-tagged (-V5) Vps34-Vps15, and beclin-1; proteins immunoprecipitated (IP) from lysates with monoclonal antibody to SLAM, as well as whole-cell lysates (WCL), were analyzed by immunoblot (IB) with antibeclin-1, anti-Vps34, anti-V5, anti-hemagglutinin, anti-SLAM or anti-EAT2. (c) Microscopy of RAW264.7 cells transiently transfected with cDNA encoding GFP-tagged beclin-1 and SLAM-mCherry, then treated with beads coated with $E$. coli outer membrane extract (at a ratio of 10:1, beads/cells) and fixed after $60 \mathrm{~min}$. Data are representative of six (a) or two (b,c) independent experiments. 